\title{
MODELLING WAVE INTERFERENCE PATTERNS USING THE SWAN MODEL
}

\author{
Gal Akrish, Delft University of Technology, G.Akrish@tudelft.nl \\ Pieter Smit, Spoondrift technologies Inc., Pieterbartsmit@gmail.com \\ Marcel Zijlema, Delft University of Technology, M.Zijlema@tudelft.nl \\ Ad Reniers, Delft University of Technology, A.J.H.M.Reniers@tudelft.nl
}

\begin{abstract}
IN-DEPTH
This study presents an efficient approach of resolving wave interference patterns in spectral wave models (e.g., SWAN). Such interference patterns, which frequently occur in coastal waters (e.g., near headlands, harbor entrances and coastal inlets), may lead to rapid changes in wave statistics, and thus, can affect wavedriven flow and transport processes. Therefore, prediction of wave conditions for coastal applications should account for these effects. Presently, operational wave models compute the mean wave properties by solving the action balance equation, which describes the transport of wave energy through geographic and spectral space, augmented with source terms to account for non-conservative and nonlinear processes. This model equation, initially intended for deep water conditions, is derived under the assumption that waves propagating at angles are mutually independent so that the wave field changes its mean properties (e.g. wave height) over many wavelengths. However in nearshore areas, the interaction of waves with variable bathymetry and currents can result in interference zones where crossing wave trains are statistically correlated and wave heights change rapidly.
\end{abstract}

In order to take into account the generation and evolution of wave interference, Smit \& Janssen (2013) have recently developed an evolution equation for what they refer to as the Coupled Mode (CM) spectrum. This new evolution equation, which is referred to as the quasi-coherent model (QCM), transports the complete second order statistics of the surface elevation. Therefore, it generalizes the action balance equation by including the evolution of the cross-correlation terms. Fortunately, the QCM can be written in the same form as the action balance equation, including a new source term that accounts for the development of wave interferences. As a result, the QCM can be readily integrated into existing spectral models.

The aim of the present study is to implement the QCM into the widely used SWAN. If successful, this would allow application of efficient spectral models to highly dynamic regions where varying bathymetry and currents lead to rapidly varying wave statistics. Specifically, we anticipate that the extended SWAN will significantly improve wave prediction in focal zones, as has been recently demonstrated by Smit et al. (2015a) for the case of propagating waves over a submerge shoal (e.g., Fig. 1). Further, although including the QCM requires additional computational effort, it need only be included in regions where interference effects might have significant influence on the wave statistics. Hence, typically the new SWAN model would behave as a conventional spectral model, with the QCM active only where needed.

However, before SWAN (or similar models) can be augmented with the QCM, several practical and theoretical issues need to be addressed. These include generalization of the QCM to account for ambient currents, inclusion of cross-correlation effects into the existing source terms and a reformulation of the QCM from wavenumber space into frequency-direction space as used in SWAN. Here, we focus on the transformation of the equations and the inclusion of coherent effects into relevant dissipative shallow water source terms (following the methodology of Smit et al, 2015b), to allow, for example, to model the diffraction effects behind patches of vegetation. We verify the model with comparison to observations and discuss efficiency and practical relevance of the resulting model for coastal applications.
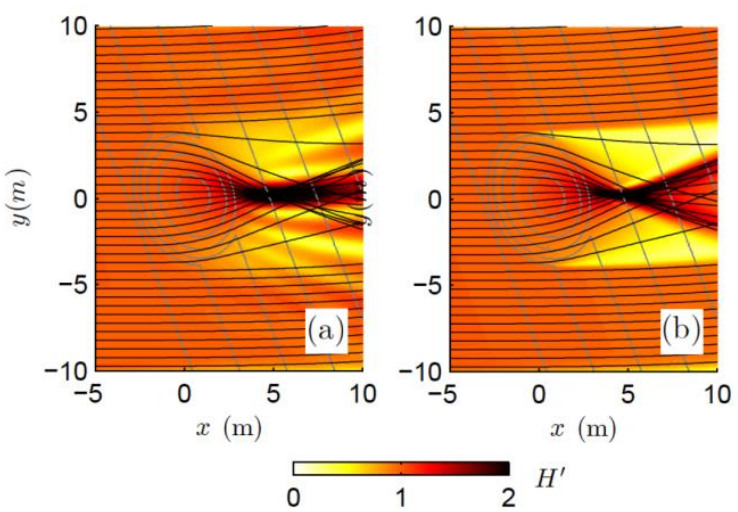

Figure 1 - Planar view of the variations in wave height for a unidirectional, monochromatic wave (incident from the left) that interacts with an elliptical shoal situated on a plane beach. Results as predicted by: (a) the QCM; (b) a conventional spectral wave model. Grey lines indicate bottom contours, while the solid black lines are wave ray trajectories. Figure adapted from Smit et al. (2015a).

\section{REFERENCES}

Smit and Janssen (2013): The evolution of inhomogeneous wave statistics through a variable medium, Journal of Physical Oceanography, vol. 43, pp. 1741-1758.

Smit, Janssen and Herbers (2015a): Stochastic modeling of coherent wave fields over variable depth, Journal of Physical Oceanography, vol. 45, pp. 1139-1154.

Smit, Janssen and Herbers (2015b): Stochastic modeling of inhomogeneous ocean waves, Ocean Modelling, vol. 96 , pp. 26-35. 